\title{
The Phenomenon of Frequency Pulling, Synchronization and Cessation of Self-oscillations of the Briggs - Rauscher Reaction Under Periodic Light Irradiation
}

\author{
D. A. Usanov, A.P.Rytik, A. V. Gagarina, O. Yu. Kutikova
}

This paper explores the phenomenon of synchronization of concentration self-oscillations of the Briggs-Rauscher reaction under external periodic exposure to white light. A high sensitivity of the self-oscillating reaction regime to periodic light irradiation is shown. The paper presents the dependence of the synchronization band on light power, establishes the limiting values of external light irradiation power at which self-oscillations "go off". The 0.04-0.1 Hz synchronization range of concentration oscillations under external light irradiation is determined. The phenomenon of frequency pulling of concentration oscillations occurred under external light irradiation with frequencies ranging from 0.029 to $0.039 \mathrm{~Hz}$ before synchronization and from 0.1 to $0.14 \mathrm{~Hz}$ after synchronization. It was established that, as the power of radiation from an external source was increased, the range of synchronization shifted to the low-frequency region of concentration oscillations, while the width of the range changed only slightly. When the luminous flux from the illumination source was less than $250 \mathrm{~lm}$, no synchronization of the self-oscillating reaction regime arose, and when the luminous flux from the source was more than $5000 \mathrm{~lm}$, the self-oscillatory process of the Briggs - Rauscher reaction ceased.

Keywords: self-oscillating reaction, synchronization, phenomenon of frequency pulling, threshold effect, oscillation phase, cessation of the self-oscillating regime

Received January 16, 2018

Accepted May 28, 2018

Dmitry A. Usanov

usanovda@info.sgu.ru

Andrei P. Rytik

ra4csz@yandex.ru

Anna V. Gagarina

av.gagarina@yandex.ru

Oksana Yu. Kutikova

oksana.296@mail.ru

Saratov State University

ul. Astrakhanskaya 83, Saratov, 410012 Russia

RUSSIAN JOURNAL OF NONLINEAR DYNAMICS, 2018, 14(2), 209-216 


\section{Introduction}

In the study of the mechanism of influence of periodic light on biological objects, in particular, on living organisms, it is of interest to consider such an influence on sensitive chemical processes, specifically, on self-oscillating chemical reactions. The latter exhibit high sensitivity to external factors [1], in particular, to the influence of light on self-oscillating reactions, one of which is the Briggs-Rauscher reaction. The self-oscillating Briggs-Rauscher reaction includes iodination and oxidation of an organic substrate (malonic acid or its derivatives) in the presence of hydrogen peroxide $\mathrm{H}_{2} \mathrm{O}_{2}$ and $\mathrm{Mn}^{2+}$ ions as a catalyst. An oscillating reaction manifests itself in a periodic change in the color of the reaction solution. In 1972, T. S. Briggs and W. C. Rauscher were the first to report on the new chemical oscillator, called "iodine clock".

In 1981, P. De Kepper and J. Boissonade [5] proposed a model describing the behavior of the self-oscillating Briggs - Rauscher reaction. The model consisted of several equations:

$$
\begin{gathered}
C \stackrel{k 1}{\longrightarrow} X, \\
2 X \stackrel{k 2}{\longrightarrow} 2 Y, \\
Y+Z \stackrel{k 3}{\longrightarrow} 2 Z, \\
X+Z \stackrel{k 4}{\longrightarrow} \text { product. }
\end{gathered}
$$

Based on kinetic equations, Boissonade obtained the following system of differential equations:

$$
\begin{aligned}
\frac{d X}{d t} & =k_{1} C-2 k_{2} X^{2}-\left(k_{4} Z+k_{E}\right), \\
\frac{d Y}{d t} & =2 k_{2} X^{2}-\left(k_{3} Z+k_{E}\right) Y, \\
\frac{d Z}{d t} & =k_{0} Y+k_{3} Y Z-\left(k_{4} X+k_{E}\right) Z .
\end{aligned}
$$

The self-oscillating Briggs - Rauscher reaction brings about changes in the concentrations of iodine $\mathrm{I}_{2}$ and iodide ions $\mathrm{I}^{-}$. The components of the reaction give first a colorless homogeneous solution, which soon turns yellow, becomes rapidly bright blue, then becomes colorless and transparent, and this process occurs again and again with a frequency of several oscillations per minute. This mixture contains hydrogen peroxide, an iodate ion, inorganic acid, malonic acid, and a catalyst - a metal ion. The Briggs-Rauscher oscillator operates well at room temperature. Oscillations occur for about 5 to 10 minutes.

The authors of [6] investigated the influence of continuous luminous flux on oscillations of the medium of the Briggs - Rauscher reaction in a stirred reactor. Three different states of the reaction were observed: excited stable, bistable, and the state of quasi-harmonic relaxation oscillations. To date, experiments involving continuous illumination, periodic illumination with different frequencies and with different duration of light pulses have been carried out. The self-oscillating regime of the chemical reaction based on the dual nature of hydrogen peroxide (Briggs-Rauscher reaction) was chosen as a sensitive test system. A description was given of the synchronization of oscillations in the Briggs-Rauscher reaction at external excitation frequencies close to the frequency of its self-excited oscillations. The authors of [8] proposed a model of synchronization of the Briggs - Rauscher reaction by means of periodic light pulses. The model proposed in [8] supplemented the well-known stages of the Briggs - Rauscher reaction with the photogeneration stage. Calculations were in qualitative agreement with the following 
experimental phenomena: change in the period and the amplitude of oscillations depending on the intensity of constant illumination; synchronization between the periodically changing illumination and the period of chemical oscillations; phase shifts induced by single light pulses, and steady states.

In [7] the results on the pattern of self-oscillations of the Belousov-Zhabotinsky reaction under exposure to light are presented. In that paper it is shown that the course of this reaction depends on the period and the amplitude of external illumination. In [9] it is described under which conditions (concentration of the initial components and power of external illumination) various regimes of oscillating structures arise in the medium of the Belousov-Zhabotinsky reaction with a negative feedback. Massive oscillations and wave patterns arose at low feedback values. Groups of clusters arose when the feedback exceeded the critical value. In addition to the standing, irregular and localized aggregates observed earlier, the authors found new types of clusters: three-phase clusters, localized irregular and localized oscillator clusters. A transition between phases was revealed, which may be due to a change in the level of the photochemical feedback.

It is important to note that the phenomena of frequency pulling and oscillation synchronization in various self-oscillating systems are observed under exposure to external light. For example, the possibility of using "frequency pulling", synchronization and breakdown of oscillations for correction of eye motion in the case of nystagmus was shown in [4]. In this paper we present results of investigation of such phenomena in the chemical self-oscillating process.

The goal of this paper is to reveal changes in the concentration oscillations of the Briggs Rauscher reaction at different parameters of periodic exposure to light. This involves revealing the most effective action parameters from the viewpoint of changes in concentration oscillations. Of particular importance is the range of variation of the frequency and power of external action. Another problem to be solved is to bring out the dependence of concentration oscillations on the length of light waves.

\section{The method of the experiment}

The self-oscillating process in the reaction medium, including changes in the amount of dissolved oxygen, was recorded using a pH-ionometer of series "Expertfrm[o]-001" with sensors: an ion-selective electrode "Yod" and a dissolved oxygen sensor (DKTP) integrated with a temperature sensor. The analysis of data was carried out using the Exp2pr program by means of fluid analyzers of series "Expertfrm[o]-001", in which the dynamics of dissolved oxygen, the electric potential of the iodine-selective electrode and the temperature in the medium is reflected in the real-time mode. Additionally, a video record was made of changes in the reaction medium in the course of the experiment. Then the chromaticity of the solution of the medium of the Briggs - Rauscher reaction was analyzed each second. The observation of periodic changes in the chromaticity made it possible to infer the frequency of chemical oscillations of the reaction medium.

During the series of experiments, which involved taking readings of the ion-selective electrode, external light was restricted to backlight, and the reaction vessel was lightproof only on the lateral side. During the video recording, constant intensity and spectral composition of illumination were maintained. Also, the temperature stabilization of the reaction medium under exposure to light was ensured. Three initial solutions prepared by the method described in detail in [2] were used. They were mixed by means of an automatic agitator. The electrodes

RUSSIAN JOURNAL OF NONLINEAR DYNAMICS, 2018, 14(2), 209-216 
which were in the reaction vessel were attached to a holder. The reaction medium was mixed originally for 5 to 7 seconds until light was turned on.

An incandescent lamp with a luminous flux of about $250 \mathrm{~lm}$ was used as a light source. It was placed under the transparent bottom of the vessel for the Briggs - Rauscher reaction (the reaction medium had a volume of $12 \mathrm{ml}$, and the liquid column was $2 \mathrm{~cm}$ in height). Illumination was regulated manually by changing the variable resistance using a timer. Checking was performed using a reaction that occurred without illumination. During constant illumination with the above-mentioned lamp, oscillations were less pronounced than during checking, and the number of oscillations was smaller, which agrees with the data presented in [2].

The analysis of the data obtained was carried out using the MS Excel 2007 program. Experiments with periodic exposure to light were conducted. The period $(T)$ had a duration of $35,30,25,20,15,10,7$, and $6 \mathrm{~s}$, which corresponded to frequencies from 0.03 to $0.14 \mathrm{~Hz}$. The relative light pulse duration was $5 \mathrm{~s}$.

It was noticed that illumination introduced an error into measurements of the electrochemical potential. To estimate this error in the readings of the electrodes, similar experiments involving exposure to light were carried out with water, into which the electrodes were submerged. It was established that the relative error of the results of such measurements is no more than $10 \%$.

Research was carried out to bring out the influence of illumination of different intensity on the Briggs - Rauscher reaction using incandescent lamps with luminous fluxes of $140 \mathrm{~lm}$ and $200 \mathrm{~lm}$ and LED lamps with luminous fluxes of $1200 \mathrm{~lm}$ and $1800 \mathrm{~lm}$ [3].

\section{Results of investigation}

Under exposure to a periodic illumination of 250-5000 $\mathrm{lm}$ and under changes of the frequency of external periodic influences from $f=0.04 \mathrm{~Hz}$ to $f=0.05 \mathrm{~Hz}$, the number of oscillations of the reactions increased by a factor of about two as compared to that during checking. One of the main conclusions drawn from the series of experiments was that in a certain frequency band the frequency of oscillations of the Briggs - Rauscher reaction was the same as the frequency of exposure to external light and that the rate of the reaction "kept up" with exposure to external light. The boundaries of the range of such a synchronization were determined.

Figure 1 shows a change in the potential in time under external periodic light irradiation with a frequency of $f=0.04 \mathrm{~Hz}$, which corresponds to the period $T=25 \mathrm{~s}$ : for $20 \mathrm{~s}$ the reaction medium was not illuminated, and for $5 \mathrm{~s}$ it was illuminated. Time is plotted in seconds along the horizontal axis, and the potential on the ion-selective electrode is plotted in millivolts along the vertical axis.

We note that after switching on the light (the velocity of increase in the luminous flux was less than 1 second) the potential on the ion-selective electrode began to increase, and after switching off the light it decreased rapidly. Such an imposition of the reaction of external oscillations with a given period became possible only in a neighborhood of the frequency of self-excited oscillations of the reaction.

Analysis of the data obtained has shown that if the period of exposure to light was somewhat smaller or larger than the period of the self-excited oscillating regime $(T=35 \mathrm{~s}, 30 \mathrm{~s}, 8 \mathrm{~s}, 7 \mathrm{~s})$, the phenomenon of frequency pulling was observed. At a frequency of exposure with a period close to the period of self-excited oscillations of the Briggs-Rauscher reaction, for example, $25 \mathrm{~s}(0.04 \mathrm{~Hz})$, with a luminous flux ranging from 250 to $23000 \mathrm{~lm}$, self-excited oscillations of 


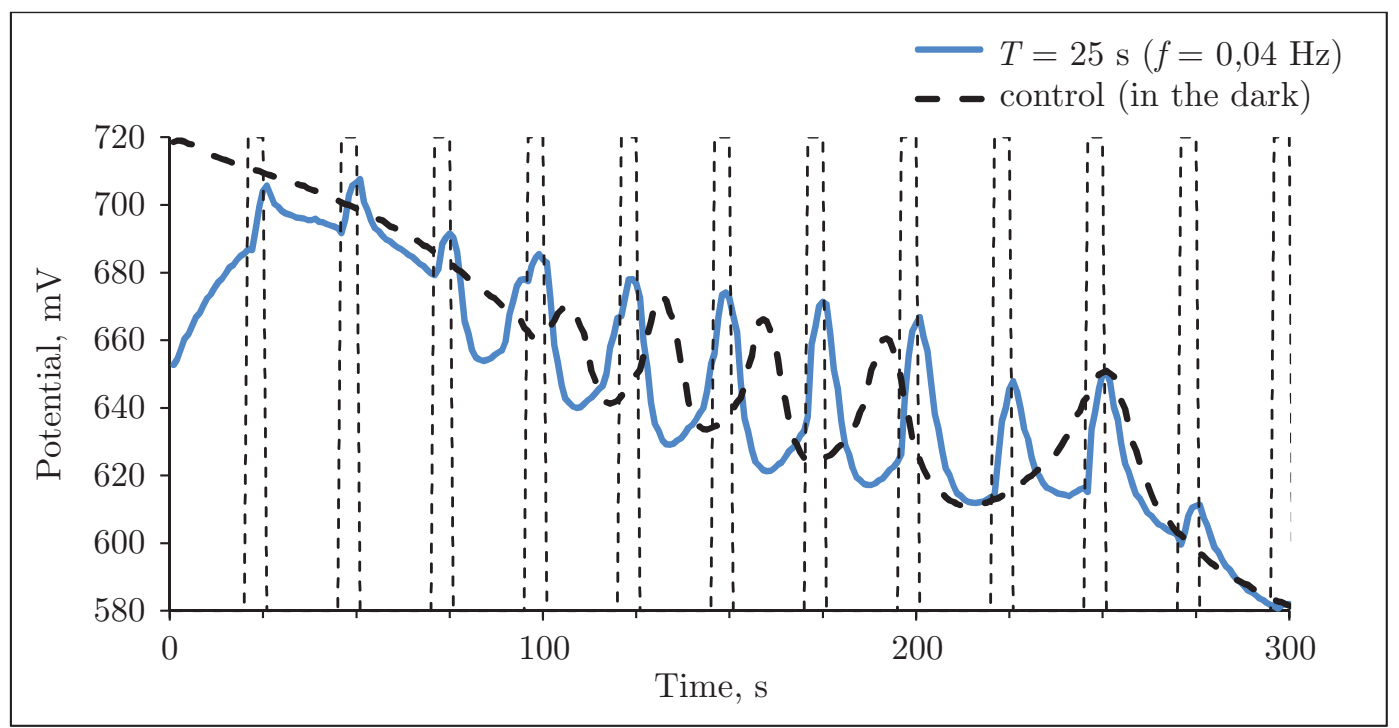

Fig. 1. Change in the potential $(\mathrm{mV})$ in time under external periodic light irradiation, $T=25 \mathrm{~s}(20 \mathrm{~s}-$ darkness, $5 \mathrm{~s}$ - light).

the Briggs - Rauscher reaction gradually synchronized with forced oscillations. For a period of external action from 30 to $10 \mathrm{~s}$ (from 0.03 to $0.1 \mathrm{~Hz}$ ) when using a lamp with a luminous flux of more than $250 \mathrm{~lm}$ the frequency of oscillations of the Briggs - Rauscher reaction became equal to the frequency of external action, that is, the synchronization phenomenon was observed. Thus, it can be concluded that the range of synchronization was found for a period of external action from 10 to 25 seconds. It can be seen from the data presented in Fig. 2 that there is a change in the frequency in the direction of exposure to light in the range $0.1-0.15 \mathrm{~Hz}$ with a luminous flux of more than $200 \mathrm{~lm}$ from the source of illumination.

Experiments were conducted to bring out the dependence of the region of synchronization on the radiation power. For this purpose, LED lamps were used in order to avoid the heating of the reaction solution. When the luminous flux was less than $250 \mathrm{~lm}$, there was no synchronization of oscillations, and only frequency pulling was observed. The results of measurements are presented in Fig. 2.

It follows from Fig. 2 that the minimal luminous flux from the incandescent lamp at which synchronization occurred in a relatively large frequency range was $250 \mathrm{~lm}$. It can be seen that the phenomenon of "frequency pulling" was observed for the following frequencies of external action with a luminous flux of $250 \mathrm{~lm}$ : 0.029; 0.033; 0.039; 0.125 and $0.143 \mathrm{~Hz}$. For the same lamp, at frequencies ranging from 0.04 to $0.10 \mathrm{~Hz}$, the phenomenon of synchronization arose, that is, the light pulse repetition frequency was the same as the frequency of oscillations of the Briggs - Rauscher reaction. For these reagents (and conditions of the experiment) the average eigenfrequency of oscillations during checking was $0.036 \mathrm{~Hz}$. When the luminous flux from the source of illumination was more than $5000 \mathrm{~lm}$, the cessation of the process of synchronization (cessation of the self-oscillating regime) was observed.

The data of Figs. 2 and 4 suggest that, as the flux of luminous radiation increased, the range of synchronization shifted to the low-frequency region, and its width changed relatively weakly. For example, under a luminous flux of $3000 \mathrm{~lm}$, synchronization started from $0.03 \mathrm{~Hz}$ and occurred approximately up to $0.07 \mathrm{~Hz}$. It should also be noted that switching on the light always initiated the yellow color of the phase of reaction, and when the light was switched off, 


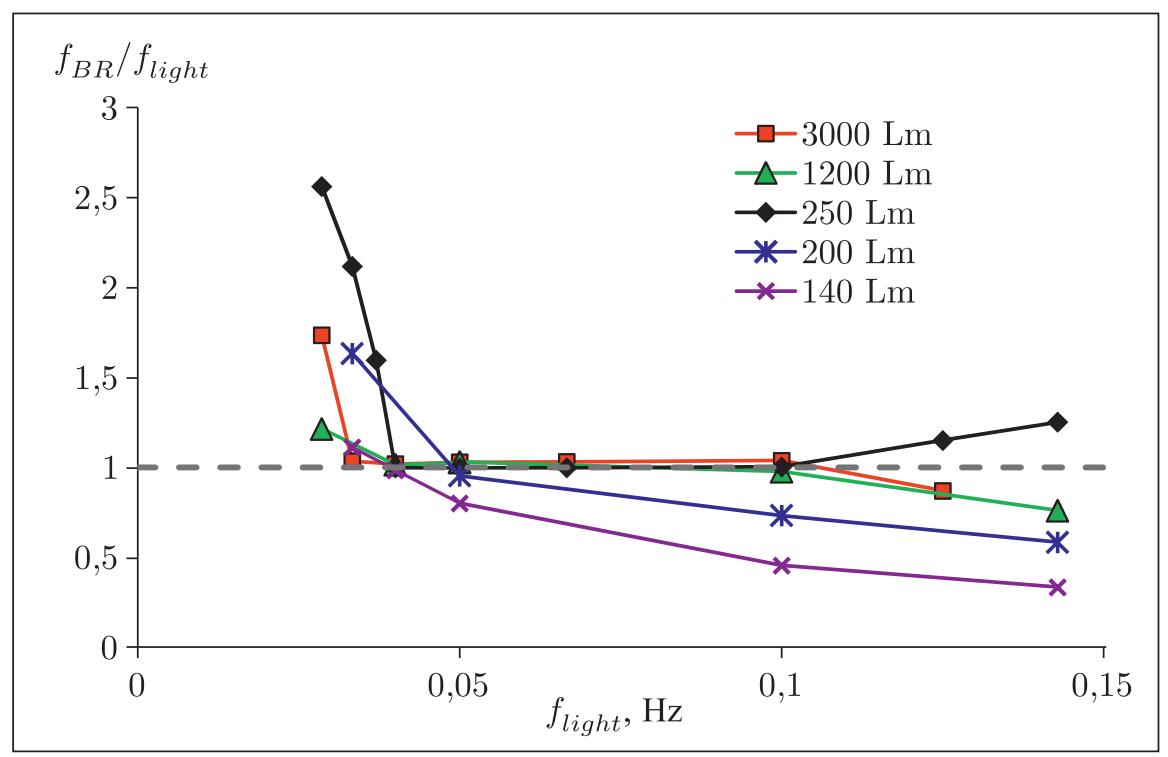

Fig. 2. Dependence of the ratio of frequencies on the frequency of illumination for different powers of radiation. The frequency of external illumination $f_{\text {light }}$ in $\mathrm{Hz}$ is plotted along the horizontal axis, and the ratio of the frequency of oscillations of the medium of the Briggs - Rauscher reaction to the frequency of external illumination $f_{B R} / f_{\text {light }}$ is plotted along the vertical axis.

the color of the solution became blue, and the larger the radiation power was, the faster the color changed. At some frequencies of periodic exposure to light, the color of the solution of the Briggs - Rauscher reaction became yellow before the light was switched on, and sharply changed from the blue to the yellow color of the phase when the light was switched on. It should be noted that, when the luminous radiation flux was more than $1200 \mathrm{~lm}$, during the last oscillations the color of the solution of the reaction began to turn blue before the light was switched off. This did not happen when the luminous flux was lower $(250 \mathrm{~lm})$ : as long as there was illumination, the reaction medium was yellow. When the luminous flux from the lamp was more than $5000 \mathrm{~lm}$, the reaction "went off", possibly due to the fact that the velocities of formation of intermediate substances were so large that the concentrations of these intermediates increased too much and the system passed from the self-oscillating regime to the stationary state (Fig. 3).

Figure 3 shows phase portraits of oscillations of the Briggs - Rauscher reaction for different frequencies of external radiation with a luminous flux of $250 \mathrm{~lm}$. The phase portraits suggest that, at frequencies close to the frequencies of self-excited oscillations $(0.037 \mathrm{~Hz}, 0.040 \mathrm{~Hz}$, $0.050 \mathrm{~Hz}$ ), there is an increase in the amplitude and the number of oscillations. It can be seen from Fig. 3 that the starting and end points of the reaction for different pulse frequencies of external illumination do almost not shift relative to each other along the ordinate axis, which is indicative of reproducibility of the initial conditions of the reaction. One can also see that, for all phase portraits, the potential differences of the electrode for the starting point (1) and the end point of the self-oscillating regime (2) differ little from each other, which suggests that the external periodic light influences only the pattern of the self-oscillating regime.

It can be seen that the rate of variation of the potential (abscissa axis) changes depending on the pulse frequency of external illumination and has a pronounced effective synchronization band. The synchronization of concentration oscillations under external light irradiation ranges from 0.04 to $0.1 \mathrm{~Hz}$. The phenomenon of pulling the frequency of concentration oscillations 

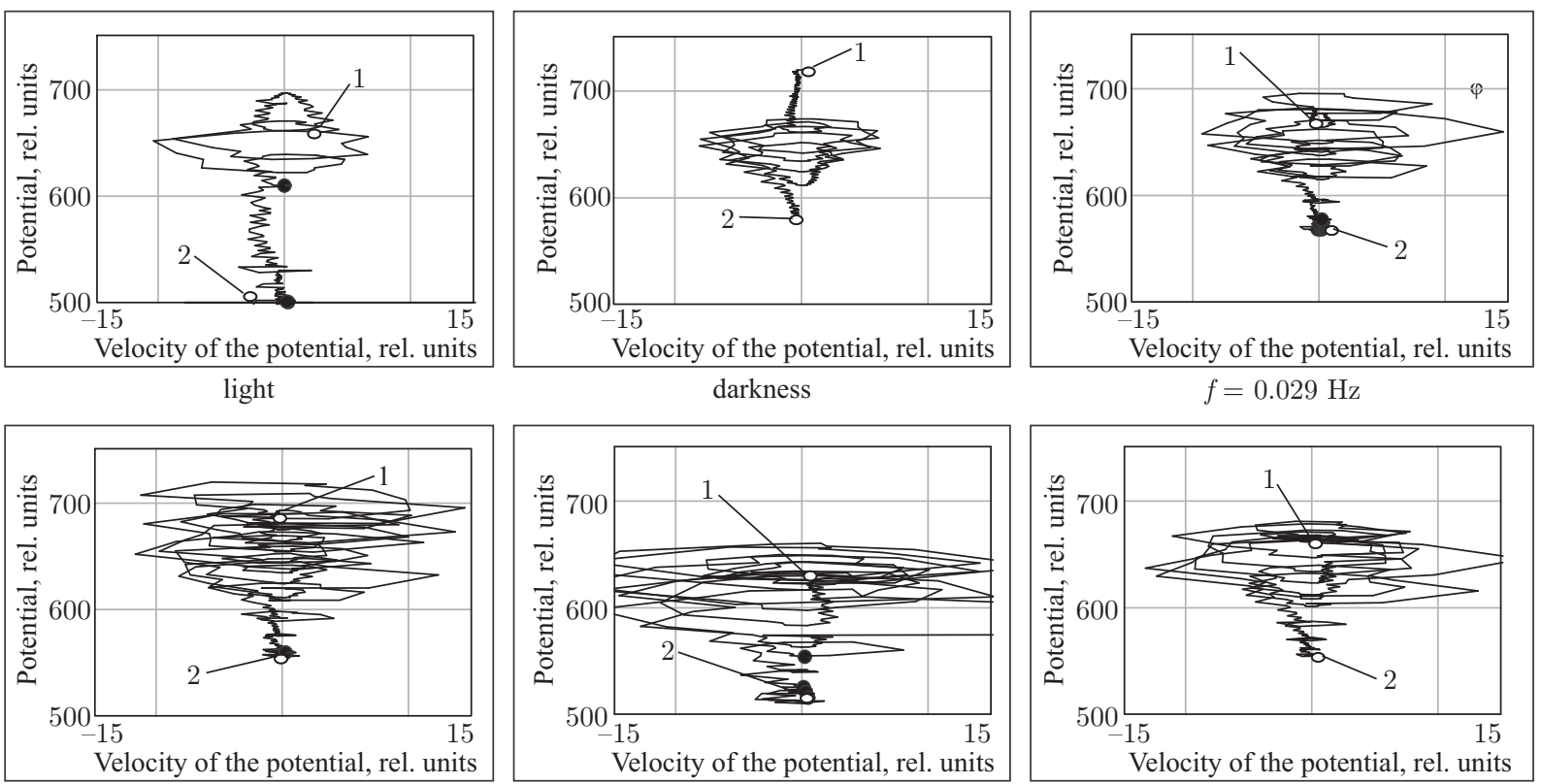

$f=0.033 \mathrm{~Hz}$

$$
f=0.037 \mathrm{~Hz}
$$

$f=0.040 \mathrm{~Hz}$
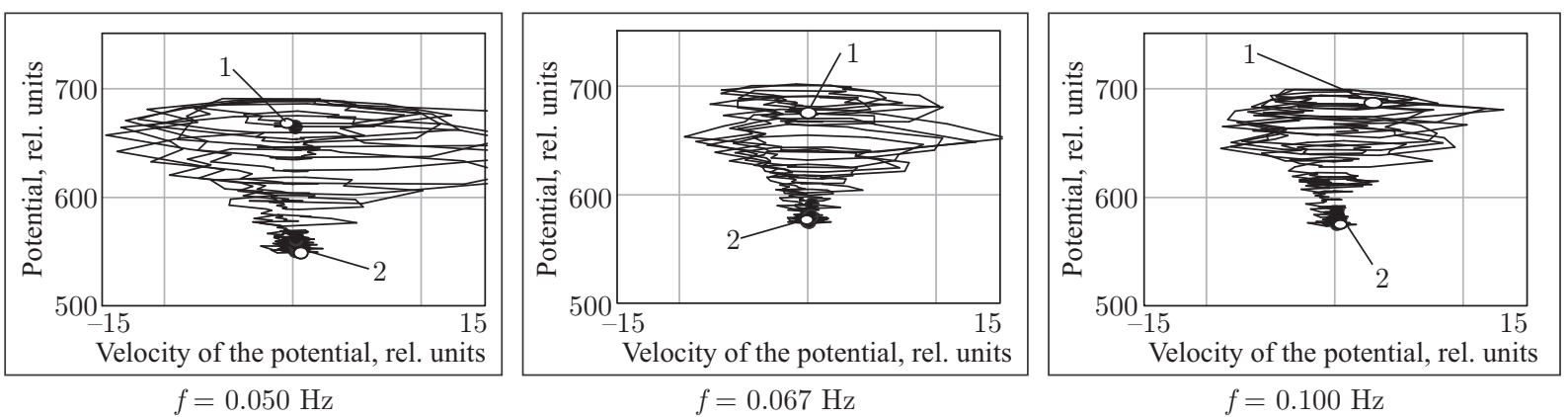

Fig. 3. Phase portraits for an illumination of $250 \mathrm{~lm}$ at frequencies $f$ from the range $0.03-0.1 \mathrm{~Hz}$. The values of the potential of the ion-selective electrode are laid off along the vertical axis, and the rate of variation of the potential (the derivative of the potential with respect to time) is laid off along the horizontal axis; 1 denotes the starting point of oscillations, and 2 denotes the end point of oscillations.

occurs under external light irradiation with frequencies ranging from 0.029 to $0.039 \mathrm{~Hz}$ before synchronization and from 0.1 to $0.14 \mathrm{~Hz}$ after synchronization. Comparison of all phase portraits shows that at a frequency of $0.037 \mathrm{~Hz}$ the rate of variation of the potential in the reaction is larger than that at other frequencies.

\section{Conclusion}

Thus, it is shown that frequency pulling, synchronization and cessation of self-oscillations can occur in a solution with the self-oscillating Briggs-Rauscher reaction as a result of periodic exposure to white light. It is demonstrated that, as the power of radiation of the external source increases, the range of synchronization shifts to the low-frequency region of concentration oscillations. It is established that the process of frequency pulling and synchronization of self-excited oscillations of the Briggs-Rauscher reaction depends on the power of the external luminous flux. Specifically, no synchronization of the self-oscillating reaction regime arose from a source 


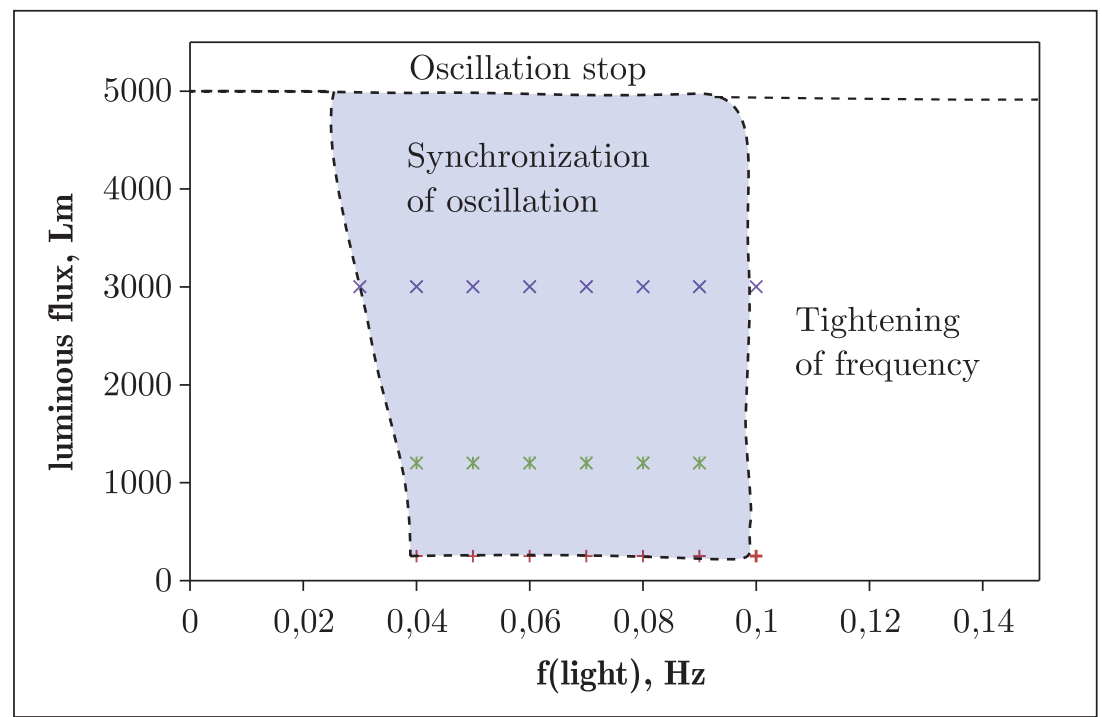

Fig. 4. Chart of regimes of oscillations of the Briggs-Rauscher reaction. The light frequency is laid off along the horizontal axis, and the light power (the corresponding luminous flux in $\mathrm{lm}$ ) is laid off along the vertical axis. Blue denotes the region of synchronization of oscillations according to the experimental data.

with a luminous flux of less than $250 \mathrm{~lm}$, while when the luminous flux from the source of illumination was more than $5000 \mathrm{~lm}$, the self-oscillating process of the Briggs-Rauscher reaction ceased. These phenomena may be of interest in investigating the influence of light irradiation on biological objects.

\section{References}

[1] Vavilin, V. A., Autocatalysis and Fluctuations in Nature, Priroda, 2005, no. 6, pp. 52-59 (Russian).

[2] De Kepper, P. and Boissonade, J., From Bistability to Sustained Oscillations in Homogeneous Chemical Systems in Flow Reactor Mode, in Oscillations and Traveling Waves in Chemical Systems, R. Field, M. Burger (Eds.), New York: Wiley-Interscience, 1985, pp. 223-256.

[3] Kozlovskaja, V. B., Radkevich, V. N., and Sacukevich, V. N., Electric Lighting: A Handbook, 2nd ed., Minsk: Tekhnoperspektiva, 2008 (Russian).

[4] Usanov, D. A., Skripal, A.V., and Usanova, T. B., Methods of Research and Correction Eye Movements in Nystagmus, Saratov: SSU, 2008 (Russian).

[5] De Kepper, P. and Epstein, I. R., Mechanistic Study of Oscillations and Bistability in the Briggs Rauscher Reaction, J. Am. Chem. Soc., 1982, vol. 104, no. 1, pp. 49-55.

[6] Dulos, E. and De Kepper, P., Experimental Study of Synchronization Phenomena under Periodic Light Irradiation of a Nonlinear Chemical System, Biophys. Chem., 1983, vol. 18, no. 3, pp. 211-223.

[7] Luo, H., Wang, C., Ren, L., Gao, Q., Pan, C., and Epstein, I. R., Light-Modulated Intermittent Wave Groups in a Diffusively Fed Reactive Gel, Angew Chem. Int. Ed., 2016, vol. 55, no. 16, pp. 4988-499.

[8] Kumpinsky, E., Epstein, I. R., and De Kepper, P., Model Study of Synchronization and Other Phenomena in Light Perturbation of the Briggs-Rauscher Reaction, Int. J. Chem. Kinet., 1985, vol. 17, no. 3, pp. 345-354.

[9] Vanag, V.K., Zhabotinsky, A. M., and Epstein, I. R., Pattern Formation in the Belousov-Zhabotinsky Reaction with Photochemical Global Feedback, J. Phys. Chem. A, 2000, no.104, no. 49, pp. 11566-11577. 